\title{
EFFECT OF ORBIT DISTORTIONS AND BETATRON TUNE ON THE RHIC POLARIZED BEAM *
}

\author{
A.U. Luccio, W.W.MacKay, F.Pilat, V. Ptitsyn, BNL, Upton, NY11973-5000, USA
}

\begin{abstract}
Polarized protons have been stored and accelerated in RHIC from $G \gamma=46.5$ to 60 during Run2000 with only one Siberian snake installed. We simulated with the spin tracking code Spink the behavior of polarized protons, in particular the effect of closed orbit distortions and betatron tune variation on the spin dynamics. According to simulation results, closed orbit and tune effects will be translated into requirements for the tune and orbit correction systems for the RHIC polarized proton Run2001, when both Siberian snakes will be available.
\end{abstract}

\section{SPINK}

The simulation of spin behavior in RHIC was done with the code Spink[1],that reads the machine lattice created by $\operatorname{Mad}[2]$. One assumption to start tracking is that in a slow process of injection and acceleration the proton spin aligns itself along the stable spin direction, that Spink finds by stroboscopic average[3] for each particle in the simulation. Multi turn tracking of many particles is done in parallel on a PC farm. In the course of tracking, machine parameters are dynamically varied to simulate real experimental conditions. For this, the code makes use of pre calculated tables and also continuously runs $\mathrm{Mad}$ in the background.

Fig. 1 shows RHIC and some of the locations that will be referred to in the following.

\section{RUN2000}

During the summer of 2000 only one Siberian snake, SNAKE1, was available on the Blue RHIC ring. The purpose of the experiment was to bring protons from injection from the AGS up to an energy corresponding to $G \gamma=60$ and measure the transverse component of the spin $S_{x}$ at the location POLAR. Up to that energy there are no strong depolarizing resonances and one snake suffices to maintain the polarization. SNAKE1 was set to produce a vertical spin flip of $\mu=180^{\circ}$ around an axis laying in the plane of the ring at an angle $\phi=13^{\circ}$ with the longitudinal coordinate. During acceleration with one snake at this setting the spin precesses around the local stable axis and must reacquire its original value when the spin tune $G \gamma$ increases by 3 units. At the diametrally symmetric location of the other missing snake, SNAKE2, the spin direction must remain longitudinal. This pattern will be distructed by the effect of spin resonances that will appear at certain values of $G \gamma$ corresponding to beats of the spin precession frequency with the vertical betatron frequency.

\footnotetext{
* Work sponsored by the U.S. Department of Energy
}

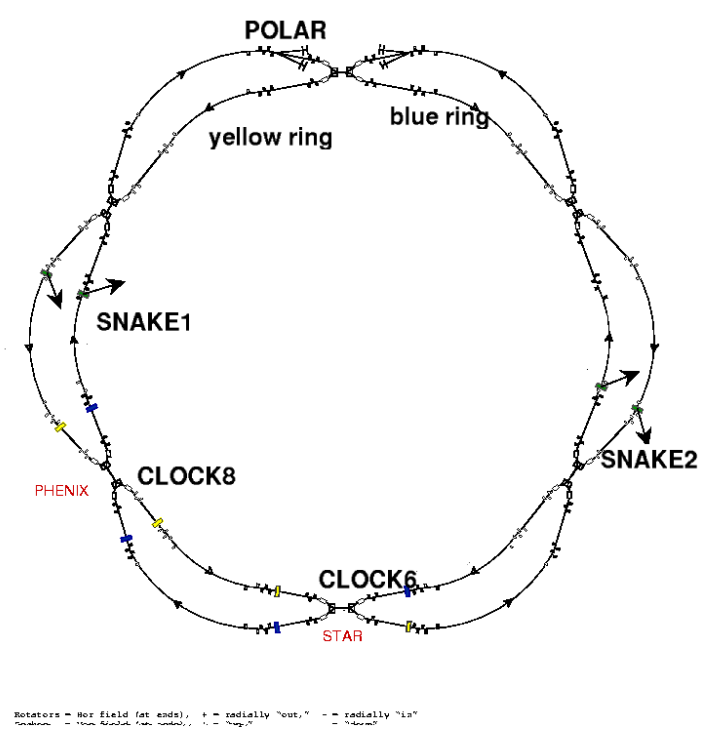

Figure 1: The Blue and Yellow rings of RHIC. Locations are for Blue

In the experiment, the lattice of the Blue ring was tuned to produce values of $\beta^{\star}=8,3,8,3,3,3 \mathrm{~m}$ in the 6 intersection regions. The measured orbit, corrected to about 3 mm was simulated in Mad using random generated errors of $0.45 \mathrm{~mm}$ rms and Micado correction with maximum corrector strength $0.37 \mathrm{mrad}$. The vertical betatron tune in the simulation was varied between $\nu_{y}=29.01$ : 29.25. Figs. 2 and 3 show results of the simulation for many cases corresponding to different values of $\nu_{y}$ in the range, for the longitudinal spin direction at SNAKE2 and the radial spin direction at POLAR, respectively. For some values of the fractional part of the tune, namely between 0.1 and 0.15 the polarization is not lost. Simulation runs were repeated for particles Gauss-randomly distributed in phase space in a $10 \pi \mathrm{mm}$-mrad $99 \%$ emittance and orbit corrected to 0.8 $\mathrm{mm}$. Results are summarized in Fig. 4, that shows the average value of the transverse polarization at $G \gamma=59$ for the two sets of runs vs. betatron tune. Comparison with experimental results are in progress; the analysis is not simple because some of the machine parameters (e.g. tune) are not known with the necessary accuracy. 

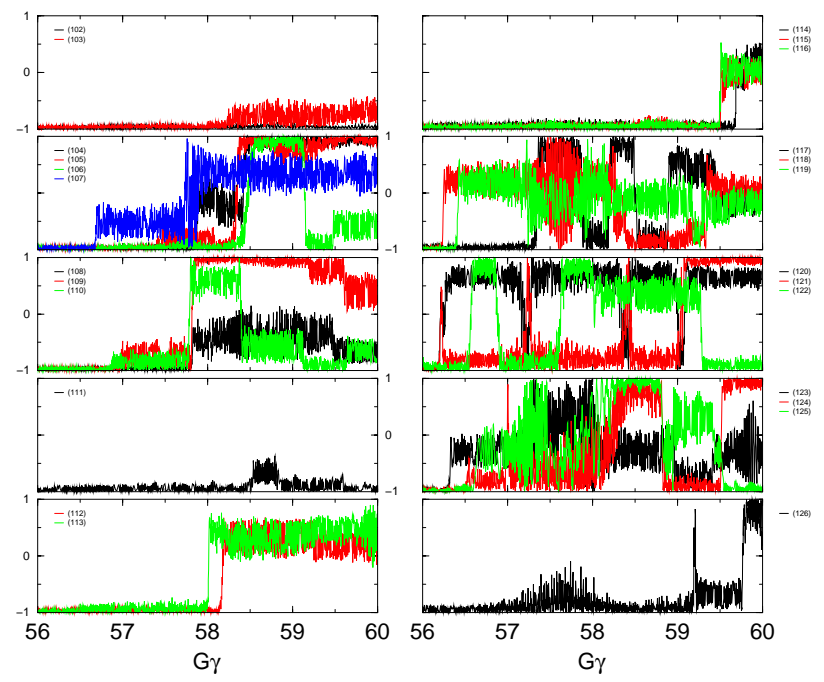

Figure 2: Longitudinal component of the spin at SNAKE2 for runs at various tunes. Orbit $2.3 \mathrm{~mm}$
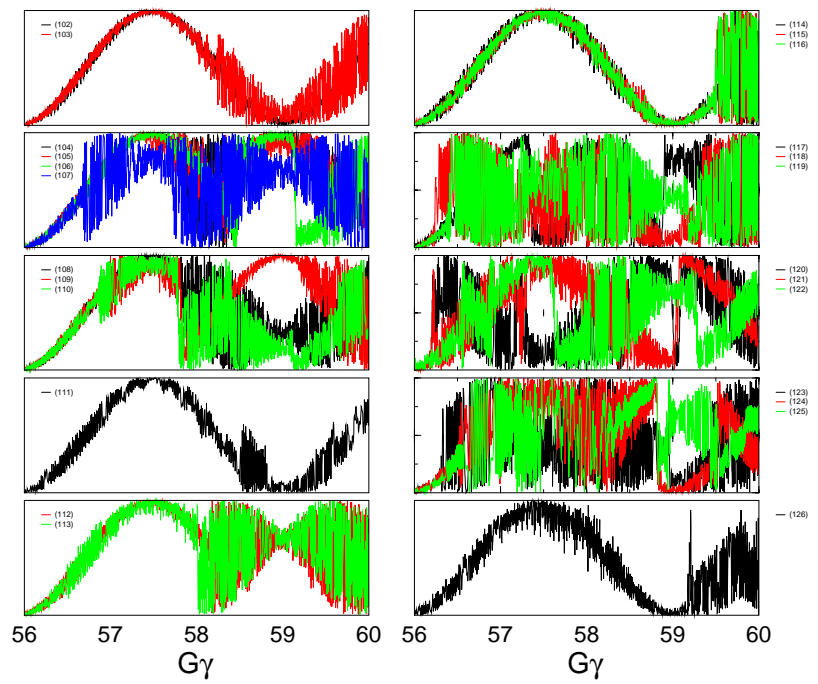

Figure 3: $S_{x}$ at POLAR for various runs

\section{RUN2001}

For the run planned for Fall 2001, both RHIC rings, Blue and Yellow, will have the full complement of two Siberian snakes installed. With two snakes it should be possible to maintain the polarization up to high proton energy. The major goal of the run is to attempt collisions of longitudinally polarized protons at locations CLOCK6 and/or CLOCK8 at a proton energy $\gamma \approx 100$, or $G \gamma=192$ or 195. The polarization will also be measured at POLAR. Since there will be no spin rotators installed in the rings yet, after having accelerated protons, we can bring the direction of the spin longitudinal at CLOCK6 and CLOCK8, and approximately longitudinal at POLAR. by adiabatically turning off one snake at constant energy[4].

Two sets of simulations were performed

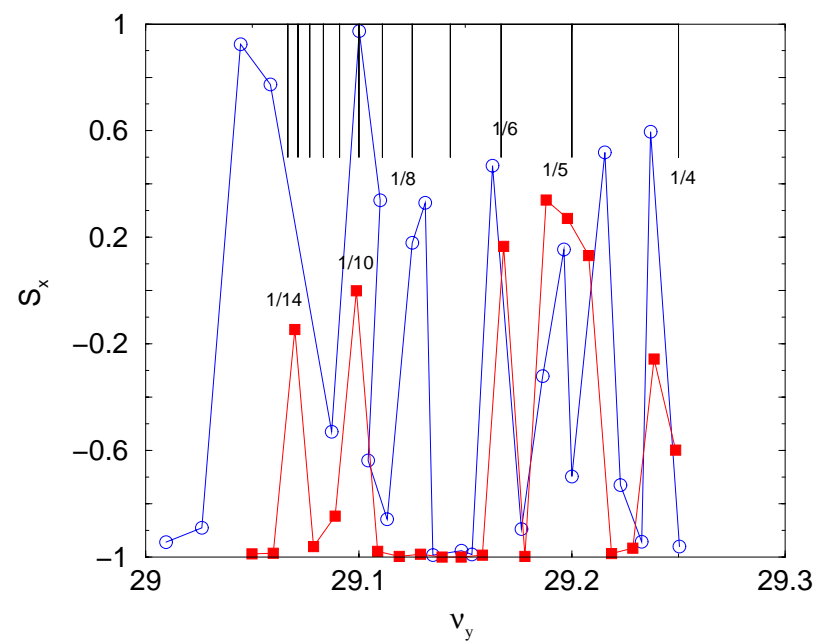

Figure 4: $S_{x}$ at POLAR for various runs. Circles are for $y_{C O D}=2.4 \mathrm{~mm}$, squares for $y_{C O D}=0.8 \mathrm{~mm}$

(i) Using a standard RHIC lattice (holy-lattice) with $\beta^{\star}=6 \times 10 \mathrm{~m}$ at all intersections, with no errors, we tracked in parallel at constant energy many polarized protons, randomly extracted on emittances of $10 \pi$ and $20 \pi$ $\mathrm{mm}$-mrad. We repeated the same game at different tunes.

(ii) We tracked on a lattice produced by Mad + Doom [5], that reads the machine, errors, closed distorted orbit (COD) and orbit corrections directly from the database containing measured values. Orbit corrections were obtained using a three-bump algorithm.

In these runs, (i) and (ii), we tracked for 60,000 turns. Since the RHIC period is $12 \mu \mathrm{sec}$, and the real operation will take possibly $600 \mathrm{sec}$, this tracking is certainly too fast, and it will be repeated later, when we will know better the machine. The spin was polarized at injection (CLOCK6) along the stable spin direction, that resulted practically vertical.

Typical values for snake angles used by Spink are shown diagrammatically in Fig. 5. An example of case (i) above is in Fig. 6. In this case, the lattice has no errors and particles being sampled lay on the circumference of an ellipse of $20 \pi$, both in $x$ and $y$. The spin is moving from the vertical to the longitudinal direction at both IR's, at it should, and to quasi-longitudinal at POLAR, that is located almost diametrally opposite to CLOCK6.

For case (ii), the real RHIC-Blue with distorted vertical COD of up to $3 \mathrm{~mm}$ is shown in Fig. 7. Tracking results are in Fig. 8, that shows $S_{z}$ at POLAR and the spin tune for all particles. This simulates a sample with $\epsilon=10 \pi \mathrm{mm}$ mrad. The tracking is rather noisy and interestingly shows two spin instabilities appearing when the spin tune reaches 0.438 , which is twice the fractional part of the vertical betatron tune of $\nu_{y}=29.219$ for this case. 


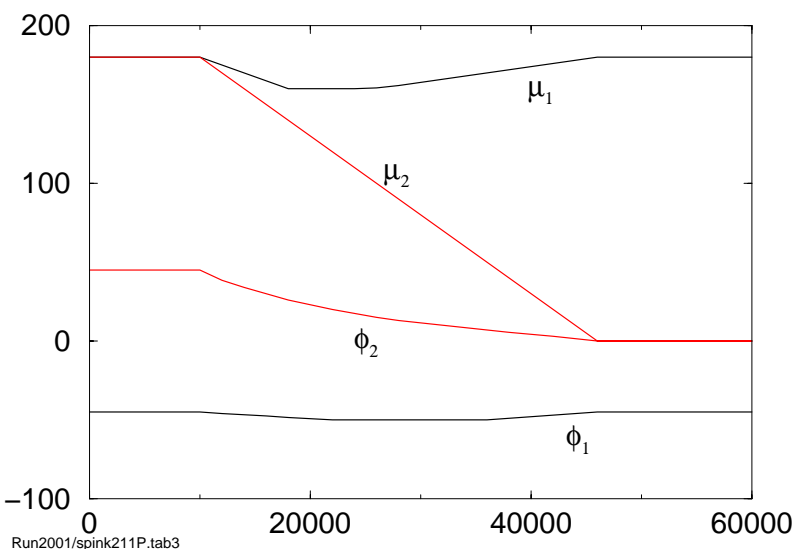

Figure 5: spin rotation $\mu$ and snake axis $\phi$ [deg], for the two snakes
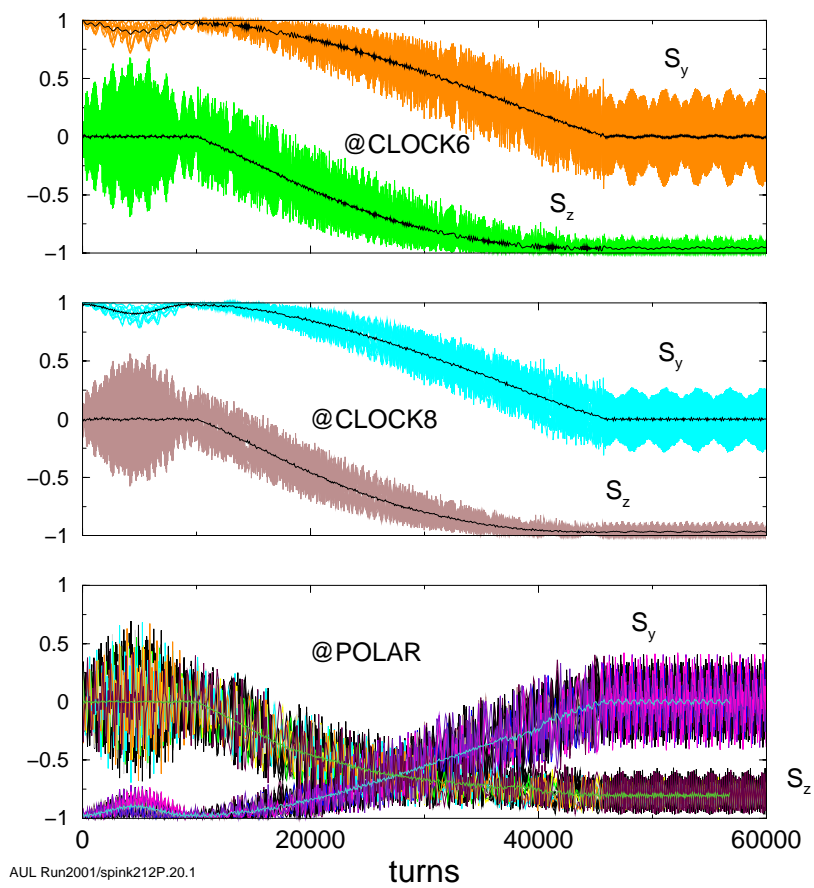

Figure 6: $S_{x}$ and $S_{z}$ at CLOCK6, CLOCK8 and POLAR. $\epsilon=20 \pi, G \gamma=192$, betatron tune $=28.208 / 29.167$

\section{ACKNOWLEDGMENTS}

Frequent discussions with T. Roser and N. Tsoupas have been essential for this work.

\section{REFERENCES}

[1] A. Luccio, in 'Trends in collider spin physics', World Scientific 1997, p.235

[2] H. Grote and F.Ch. Iselin, 'The Mad Program, vers. 8.19', CERN/SL/90-13, Geneva 1996

[3] K.Heinemann and G.H.Hoffstätter, Phys. Rev. E54, 4240,1996

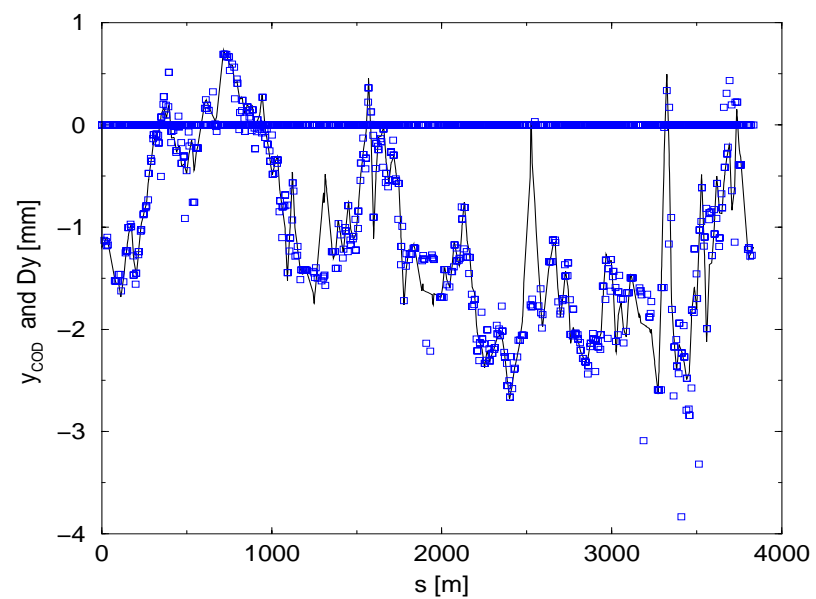

Figure 7: $y_{C O D}$ (solid line) and vertical quad errors (o)

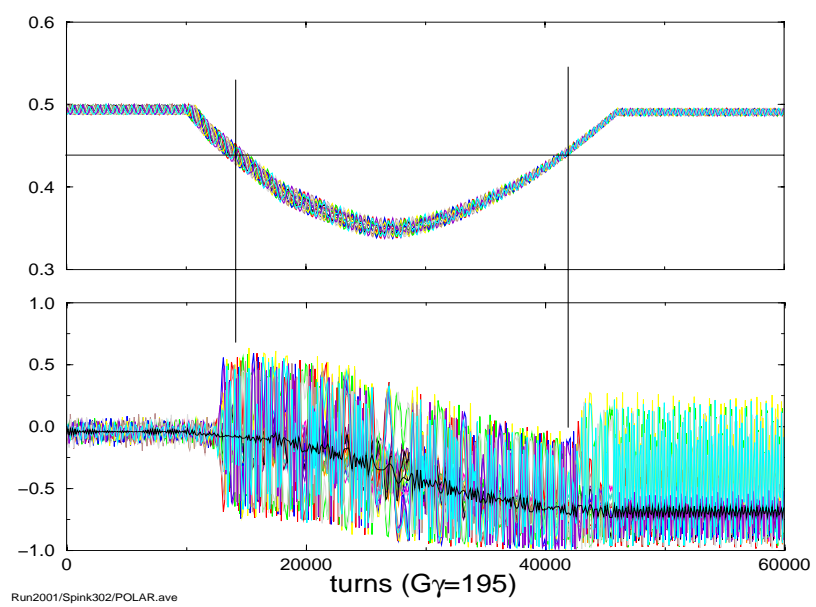

Figure 8: Spin tune and longitudinal component of the spin, $S_{z}$ at POLAR. $G \gamma=195$

[4] W.W.MacKay et al. 'Commissioning and Future Plans for Polarized Protons in RHIC', these Prooceedings

[5] CERN, Accelerator Physics Group, 'The Doom Project', http://wwwslap.cern.ch/act/doom 